Revue d'histoire de l'Amérique française

REVUE D.HISTOIRE DE L'AMÉRIQUE FRANÇAISE

\title{
Une étude de mentalité : les injures verbales au Canada au XVIII $^{\mathrm{e}}$ siècle (1712-1748)
}

André Lachance

Volume 31, numéro 2, septembre 1977

URI : https://id.erudit.org/iderudit/303609ar

DOI : https://doi.org/10.7202/303609ar

Aller au sommaire du numéro

Éditeur(s)

Institut d'histoire de l'Amérique française

ISSN

0035-2357 (imprimé)

1492-1383 (numérique)

Découvrir la revue

Citer cette note

Lachance, A. (1977). Une étude de mentalité : les injures verbales au Canada au $\mathrm{XVIII}{ }^{\mathrm{e}}$ siècle (1712-1748). Revue d'histoire de l'Amérique française, 31(2),

229-238. https://doi.org/10.7202/303609ar d'utilisation que vous pouvez consulter en ligne.

https://apropos.erudit.org/fr/usagers/politique-dutilisation/ 
NOTES DE RECHERCHE

\title{
$-\mathrm{I}-$ \\ UNE ÉTUDE DE MENTALITÉ: LES INJURES VERBALES AU CANADA AU XVIII' SIÈCLE (1712-1748)*
}

\author{
ANDRÉ LACHANCE \\ Département d'bistoire \\ Université de Sherbrooke
}

L'étude des injures verbales ${ }^{1}$, c'est-à-dire de toutes paroles qui portent directement ou indirectement atteintes à l'honneur d'autrui ${ }^{2}$, peut être révélatrice de la mentalité d'une société donnée. Par les injures verbales une société se définit par opposition. "Le vocabulaire négatif de l'injure si l'on ne se contente pas de le recenser en le déracinant... déborde largement l'intérêt philologique car il découvre, écrit Yves Castan, cette guerre intestine qu'une civilisation mène toujours contre ses abus et ses archaïsmes, ses hérésies et ses périls, par laquelle aussi elle cherche à se délivrer de ses remords et de ses angoisses ${ }^{3}$. De plus l'injure verbale pour amener une réprobation irréfutable, insoutenable, sur l'injurié, doit percuter et être ressentie par celui-ci comme quelque chose de vraisemblable et de rattachable à sa personne. C'est dans ce sens que l'insulte est un instrument

* Version remaniée d'un texte présenté à la réunion de la Société historique du Canada (Frédériction, juin 1977), en guise de commentaire à une communication de Peter N. Moogk, intitulée "Insults, Epithets and Popular Psychology in New France». M. Moogk a établi son étude sur 135 cas de "plaintes en réparation d'injure verbale" entendus devant les tribunaux de Montréal, Trois-Rivières, Québec et Louisbourg entre 1658 et 1760 soit sur uniquement $46 \%$ environ de toutes les affaires de ce genre traduites devant les cours de justice royale.

1 Cette analyse des injures verbales doit être replacée dans le cadre d'une étude plus vaste que nous avons menée sur la criminalité au Canada entre 1712 et 1748. Voir ma thèse de doctorat «La justice criminelle du roi au Canada, 17121748» (Université d'Ottawa, 1974), 72-119, 187-248, 294-337. I: 2 et 8 .

2 F. Dareau, Traité des injures dans l'ordre judiciaire (2 vols, Paris, 1785),

3 Y. Castan, Honnêteté et relations sociales en Languedoc, 1715-1780 (Paris, Plon, 1974), 42.

RHAF, vol. 31, n 2 (septembre 1977) 
d'analyse éclairant pour étudier la mentalité du Canada au XVIIIe siècle. En nous basant sur les 106 procès (soit près de 20\% (19.8\%) des 534 procès criminels que nous avons retrouvés entre 1712 et 1748) pour injure, calomnie, médisance, diffamation et injure et voie de fait retrouvés dans les fonds judiciaires des archives de Québec, Montréal et Trois-Rivières, nous avons mené une analyse de ce langage ultime qu'utilisent les Canadiens dans une situation de crise. La mentalité de la société canadienne transparaît principalement à travers les «informations», les témoignages, les interrogatoires des inculpés. En effet, plaignants, témoins et accusés doivent dessiner en traits pleins devant la cour leurs indignations, leurs réactions ou leurs justifications que le greffier transcrit dans ses registres avec une précision toute juridique. L'important ici est de pousser l'étude plus loin que ce qu'un simple recensement comme celui de Robert-Lionel Séguin dans L'injure en Nouvelle-France ${ }^{4}$ peut nous révéler. Contrairement à ce qu'a fait M. Séguin, il faut étudier les injures verbales en fonction de ses usagers et de leurs situations et s'interroger sur les motifs qui poussent les Canadiens à proférer ces insultes. Seul l'étude du vocabulaire négatif de l'injure replacée dans son contexte nous permet de mieux percevoir la société canadienne. Certes l'image de la société que les injures verbales nous présentent n'est qu'un reflet partiel de celle-ci car les injures que l'on retrouve devant les tribunaux ne sont qu'une infime portion de la criminalité réelle. Il existe un écart important sous l'Ancien Régime entre les causes qui donnent lieu à des poursuites et les affaires qui ne viendront jamais devant les cours de justice et qui, par conséquent, nous échappent. Toutefois, même si nous ne pouvons connaître le réel avec précision, nous croyons que les données que nous avons offrent un intérêt certain pour mieux cerner le probable.

Les injures verbales s'inscrivent dans la banalité du quotidien au Canada. Elles nous montrent une société active et pleine de vie où les contacts humains sont intenses. Le campagnard y apparait comme très attaché à ses biens, à ses droits et à son code d'honneur. Les dégâts causés par les divagations des animaux dans les champs des voisins sont à l'origine de plusieurs altercations. Au printemps de 1744 à Verchères, une dispute éclate entre les voisins Michel Privé, marchand, et Nicolas Bissonnette, habitant; lorsque Privé, dont le cheval est allé sur la terre de Bissonnette manger du grain, refuse de payer les dégâts causés par son cheval, on se traite de

4 (Montréal, Leméac, 1976), 250-(2). 
"voleur, fripon, coquin ${ }^{5}$. À d'autres moments, on se dispute entre voisins pour des questions de prêts ${ }^{6}$, de clôture $^{7}$, de creusage d'un fossé $^{8}$ et de droit de passage sur le chemin public. Ainsi, en février 1734, sur le chemin de la côte Saint-Michel, Louis Langlois dit Traversy, fermier de Thérèse de Couagne, veuve du Sieur Poulin de Francheville, refuse de céder le chemin au fermier de Monsieur Dubuisson, Jean Mailhot dit Latulipe, comme si c'était déchoir que de lui permettre de le dépasser ${ }^{9}$. À ce refus de Langlois est lié tout un schème de représentations sociales. Et les animosités entre voisins peuvent être tellement graves que l'on est obligé quelquefois de faire appel au curé pour pacifier les esprits. Au printemps de 1713, à l'île Jésus, le curé pour ramener la paix entre Jean Taillon et André Colin dut intervenir auprès d'eux bannière et croix à la main ${ }^{10}$.

Quant au citadin, en dehors des heures de travail, c'est au cabaret ou dans la rue que nous le retrouvons. Au cabaret, il s'y réunit avec des amis autour d'une table pour boire, chanter, jouer aux cartes (les jeux de cartes à la mode sont «la Triomphe» et le «Pharaon») ou au billard. Le vin qu'on y boit et le jeu qu'on y pratique sont fréquemment à l'origine de tensions qui s'expriment par des injures et dégénèrent en bataille que l'on pourrait qualifier quelquefois de cruelle. À Québec, le 4 avril 1714, le cordonnier Louis Rousseau disputait une partie de billard avec son collègue cordonnier Joseph Dugas. À la suite d'un jeu de Rousseau, celui-ci fut accusé par son compagnon d'avoir triché. Des mots aigre-doux, on passa aux insultes puis aux coups. On devint à ce point furieux que les deux joueurs se sautèrent dessus, se roulèrent par terre et se mordirent à qui mieux-mieux. Louis Rousseau coupa avec ses dents deux doigts de Joseph Dugas alors que ce dernier mordit à pleines dents dans la joue de son adversaire lui en arrachant même un morceau ${ }^{11}$.

Dans la rue, l'activité y est aussi intense; on y parle entre passants, entre voisins, des événements qui font la «manchette». À

au 19.

5 ANQM, Documents judiciaires, feuillets séparés, janvier-juin 1744, 3 juin

6 ANQM, ibid., octobre 1713, mars-avril 1714, décembre 1714, septembre 1715. juillet 1723; ANQQ, NF 25, PJN 483 1/2.

7 ANQM, ibid., août 1728.

8 ANQM, ibid., octobre 1725 , août 1727 , novembre-décembre 1728.

9 ANQM, ibid., janvier-mars 1734 et avril-juin 1734, 10 février au 24 mai.

10 ANQM, ibid., 9 au 31 octobre 1713.

11 ANQQ, Matières de Police, 1695-1755, ff. 65-94; ANQM, Documents judiciaires, janvier-juin 1720,22 janvier, 18 mars. 
la fin de mai 1735, la guerre des Renards occupait les esprits, le marchand montréalais Julien Trottier Desrivières et René Godefroy de Linctôt en discutaient lorsque les choses s'enflammèrent et que l'on commença à se traiter de «sot» et de «chien» ${ }^{12}$. En d'autres occasions, c'est à la suite d'une simple bousculade que les insultes fusent. En décembre 1728, à Montréal, Joseph Saulquin dit SaintJoseph, soldat travaillant aux magasins du roi, se promenait rue Saint-Paul lorsque Jacques Jousselan dit L'Africain, maître maçon, passant près de lui, lui donna un coup de coude et murmura «Tu es un bougre de coquin». Saulquin répliqua aussitôt en assénant à Jousselan plusieurs coups de canne et de poing ${ }^{13}$. Des taquineries peuvent, à l'occasion, susciter la colère et les injures verbales. Le 5 février 1731, le maçon Charles Pépin, «pris de vin» (en état d'ébriété), - il fêtait à sa façon le lundi gras - passa devant la maison du cordonnier montréalais Michel Barré et s'arrêta pour y satisfaire ses besoins naturels. Devant ce spectacle, Barré, qui se tenait devant la porte de sa demeure, pour le taquiner, lui dit: «Oh que voilà un joly bougre qui pisse bien.» Ces paroles ne semblèrent pas piaire à Pépin qui se jeta sur le cordonnier en le traitant de «bougre de cochon ${ }^{14}$.

Le vocabulaire que les Canadiens utilisent dans ces moments de crise est un indicateur précieux pour connaitre les comportements sociaux répréhensibles et les valeurs véhiculées (voir tableau I). Selon que l'on s'adresse à des femmes qui constituent plus du tiers $(38,7 \%)$ de toutes les victimes pour insultes au $\mathrm{XVIII}^{\mathrm{e}}$ siècle (voir tableau II) ou à des hommes, les injures varient. Les mots utilisés pour insulter une femme au Canada font surtout appel à son code d'honneur. Pour la femme, l'honneur est considéré comme un dépôt qu'elle a en elle, qu'elle doit défendre et dont elle doit rendre compte à ses parents, à son mari et à ses enfants. Cet honneur se résume principalement à la chasteté car dans la société d'Ancien Régime, il est entendu que la femme doit réserver à son mari et à ses enfants toutes ses puissances d'affection et de fécondité. C'est pourquoi à cette époque le crime le plus honteux pour elle est celui de la prostitution. Par conséquent, l'insulte qu'elle considère comme la plus grave et qui la touche davantage est celle de "putain» et ses synonymes «garce», «macrelle»,

12 ANQM, ibid., mai-juin 1735, 1er au 11 juin 1735.

ANQM, ibid., 30 et 31 décembre 1728,1 er, 14 et 18 janvier 1729 .

ANQM, ibid., janvier-avril 1731, 6 au 17 février 1731. 


\section{TABLEAU I}

Fréquence d'utilisation des injures

verbales au Canada au XVIII ${ }^{e}$ siècle (1712-1748)

Fréquence

(Nbre de fois retrouvée)

Homme Fréquence Femme Fréquence Homme ou Totaux

\begin{tabular}{|c|c|c|c|c|c|c|}
\hline 20 & Fripon & 4 & Friponne & - & - & 24 \\
\hline 10 & Voleur & 2 & Voleuse & - & - & 12 \\
\hline 6 & Coquin & - & - & - & - & 6 \\
\hline 3 & Gueux & 3 & Gueuse & - & - & 6 \\
\hline 2 & Maraud & - & - & - & - & 2 \\
\hline 1 & Receleur & - & - & - & - & 1 \\
\hline 1 & Faquin & - & - & - & - & 1 \\
\hline 1 & Canaille & - & - & - & - & 1 \\
\hline 1 & «Cartouche» & - & - & - & - & 1 \\
\hline - & - & 18 & Putain & - & - & 18 \\
\hline- & - & 6 & Garce & - & - & 6 \\
\hline - & - & 1 & Macrelle & - & - & 1 \\
\hline - & - & 1 & $\begin{array}{l}\text { Coureuse de } \\
\text { garçons }\end{array}$ & - & - & 1 \\
\hline 1 & $\begin{array}{l}\text { Receleur de } \\
\text { bordel }\end{array}$ & - & - & - & - & 1 \\
\hline 20 & Bougre (a) & 11 & Bougresse (a) & - & - & 31 \\
\hline 3 & Sot & - & - & - & - & 3 \\
\hline - & - & - & Folle & - & - & $1=$ \\
\hline 2 & Chien & - & - & - & - & $2^{-7}$ \\
\hline 1 & Cochon & - & - & - & - & 1 \\
\hline- & - & 1 & Élan & - & - & 1 \\
\hline- & - & 1 & Cavale & - & - & 1 \\
\hline- & - & - & - & 3 & $\begin{array}{l}\text { Qualifica- } \\
\text { tif + bête }\end{array}$ & 3 \\
\hline 1 & Insolent & 1 & Insolente & - & - & $2^{-}$ \\
\hline 4 & Foutre & - & - & - & - & 4 \\
\hline 1 & Sorcier & - & - & - & - & 1 \\
\hline 1 & Crasseux & - & - & - & - & 1 \\
\hline 1 & Laquais & - & - & - & - & 1 \\
\hline- & - & 1 & $\begin{array}{l}\text { Femme de } \\
\text { panis }\end{array}$ & - & - & 1 \\
\hline- & - & - & - & 3 & $\begin{array}{l}\text { Repris de } \\
\text { justice }\end{array}$ & 3 \\
\hline 1 & $\begin{array}{l}\text { Fils de bour- } \\
\text { reau }\end{array}$ & 1 & $\begin{array}{l}\text { Fille de } \\
\text { bourreau }\end{array}$ & - & - & 2 \\
\hline- & - & - & - & 2 & $\begin{array}{l}\text { Reste de } \\
\text { gibet }\end{array}$ & 2 \\
\hline- & - & - & - & 1 & $\begin{array}{l}\text { Gens de } \\
\text { sac et de } \\
\text { corde }\end{array}$ & 1 \\
\hline - & - & - & - & 1 & $\begin{array}{l}\text { Race de } \\
\text { pendu }\end{array}$ & 1 \\
\hline - & - & - & - & 1 & $\begin{array}{l}\text { Race de } \\
\text { «Darles» }\end{array}$ & 1 \\
\hline
\end{tabular}

(a) Ce terme est habituellement utilisé avec d'autres insultes comme une sorte de qualificatif devant l'injure principale. 
TABLEAU II

Sexe

\begin{tabular}{|c|c|c|c|c|c|c|c|c|c|}
\hline \multicolumn{4}{|c|}{ Accusés } & \multirow{3}{*}{$\begin{array}{c}\text { Total } \\
145\end{array}$} & \multirow{2}{*}{\multicolumn{2}{|c|}{ M \% }} & \multicolumn{3}{|c|}{ Victimes } \\
\hline & $\%$ & & & & & & & & Total \\
\hline 106 & 73.1 & 39 & 26.9 & & 87 & 61.2 & 55 & 38.7 & 142 \\
\hline
\end{tabular}

«coureuse de garçons». Nous avons constaté que ce genre d'injure était la plus répandue ( 27 fois) au XVIII ${ }^{\mathrm{e}}$ siècle. Parmi toutes les femmes qui, au XVIII ${ }^{\mathrm{e}}$ siècle, eurent leur honneur blessé de la sorte, Catherine Trottier, femme de François Bellestre, officier dans les troupes de la Marine, reçut les insultes les plus infamantes lorsque Charlotte Turpin, femme de Simon Réaume, fermier des Bellestre, la traita de «gueuse, élan, garce» et surtout de «plus putain qu'une cavale ${ }^{15}$. Or, lorsque l'on sait qu'une cavale est une jument qui sert à la reproduction, il ne peut $\mathrm{y}$ avoir d'injures plus déshonorantes. On lance aussi aux femmes des insultes comme "putain publique», "putain d'ivrognesse», «fausse putain de ménagère», «coureuse de garçons», bougre de gueuse», etc... La femme se rend également devant les tribunaux pour rétablir son honneur qu'une diffamation a terni. En juillet 1714, Henry Delaunay, maître charron, se présenta devant Rouer D'Artigny, juge à la prévôté de Québec pour demander que l'honneur et la réputation de sa fille Barbe soient «réparés», car Jean-Baptiste La Grange dit Toulouse, domestique à l'Hôtel-Dieu de Québec, avait déclaré publiquement qu'il avait vu Barbe Delaunay avec un homme dans un champ, «dans une posture indécente et en train de commettre le crime de paillardise ${ }^{16}$. En avril 1721, c'est Louis Dragon dit Guay, habitant de Boucherville, père de Louise, qui à son tour demanda au tribunal montréalais une réparation d'honneur pour sa fille. Mathurin Grégoire, habitant de Contrecœur, avait dit à plusieurs personnes qu'il avait eu par deux fois «un commerce charnel» avec la fille de Guay «en épiant, pour jouir de la fille, déclarait-il, le temps où les parents n'y étaient pas ${ }^{17}$. Bref, toutes ces injures traduisent un souci très pointilleux de la légitimité sexuelle, la même victime du tourbillon oratoire

15 ANQM, ibid., juillet-décembre 1728, 9 et 10 novembre, 14 décembre, janvier-avril 1729, 14 janvier.

16 ANQQ, NF 13-1, Matières de Police, 1695-1755, f. 100 ss. ANQM, Documents judiciaires, janvier-juin 1721, 7 juin.

17 ANQQ, NF 21-15, Documents de juridiction de Montréal, 1676-1723, 29 avril 1721 au 15 juillet 1722 . 
pouvant devenir, au gré de l'inspiration, l'auteur ou la victime de pratiques sexuelles que l'on considère comme condamnables. Ces insultes sont d'ailleurs toujours utilisées en défaveur de la victime et comme significatives de l'ignominie de ses mœurs. Ainsi pour les femmes, la prostitution évidente ou imaginaire leur est reprochée avec beaucoup de vigueur et souvent par ceux qui tirent parti du relâchement de leurs mœurs.

Pour les hommes, l'accusation la plus infamante est celle qui attaque son honnêteté et par conséquent l'injure la plus fréquente (45 fois) et la plus atroce qui les blesse davantage est celle de «fripon» (20 fois) et ses synonymes «voleur» (10 fois), «coquin» (6 fois), «gueux» (3 fois), «maraud» ( 2 fois), «receleur» (1 fois), «faquin» (1 fois), «canaille» (1 fois) et «cartouche» ( 1 fois). En octobre 1734, à Montréal, Jean Bouchard dit Lavallée, maître forgeron, se rendit chez Jacques Lecavalier, maitre arquebusier. Il était irrité par le fait que Lecavalier avait mis en prison son compagnon de boutique, François Rainville, parce qu'il n'avait pas encore payé les 51 livres qu'il lui devait ${ }^{18}$. Le forgeron demanda donc à l'arquebusier s'il considérait Rainville comme un fripon pour l'avoir ainsi fait emprisonner. Lecavalier lui répondit que Rainville était un «crasseux» comme lui. C'est alors que Jean Bouchard dit Lavallée, au paroxysme de la colère, traita l'arquebusier de «bougre de fripon», de "voleur» ${ }^{19}$. Pour les hommes comme pour les femmes, l'honneur est un bien précieux sur lequel ils veillent. Être accusé de parent de l'exécuteur de la haute justice ou d'un repris de justice était considéré à l'époque comme une atteinte grave à son honneur et à sa réputation, car dans la société canadienne comme dans la société française d'Ancien Régime, le déshonneur (l'infamie) était lié à la condition de bourreau comme à celle des criminels que le maître des hautes œuvres exécutaient. Jean-François Fournel, avocat au Parlement de Paris au XVIII ${ }^{\mathrm{e}}$ siècle, écrivait: "S'il y a un état dans le monde qui semble dévoué de droit à l'infamie et à l'exécration générale, c'est sans contredit celui de Bourreau ${ }^{20}$.»En mars

18 Il s'agit d'un des deux cas d'emprisonnement pour dettes que nous avons retrouvés pour cette période. L'autre incarcération est celle d'un nommé Nouette qui doit 400 livres aux marchands Jean-Mathieu Mounier et Pierre Jéhanne (ANQQ, NF 25, PJN 1394).

19 ANQM, Documents judiciaires, juillet-décembre 1734, 27 octobre au 3 novembre

20 F. Dareau, op. cit., I: 80, note 23. 
1737, le navigateur Laurent Roy doit se rétracter publiquement pour avoir déclaré que Philippe Benoît, aubergiste à Québec, était le «fils d'un maître des hautes œuvres des environs de la ville de La Rochelle ${ }^{21}$. Ce type de diffamation était assez souvent utilisé (10 fois) au XVIII ${ }^{e}$ siècle au Canada. On se traite aussi de «race de pendu», "repris de justice», "reste de gibet», "gens de sac et de corde» comme aussi de «sorcier». Á ce propos, bien qu'au $\mathrm{XVIII}^{\mathrm{e}}$ siècle nous ne retrouvions pratiquement plus de cas de sorcellerie devant les tribunaux, celui qui se fait accuser de «sorcier» se considère toujours blessé dans son honneur et exige une réparation publique. En juin 1724, un habitant de la côte Saint-Joseph, Mathurin Duroques, fut qualifié de «sorcier» par Gilles Lauzon, habitant, parce que la vache de Lauzon dont Duroques avait têté le lait, tomba malade par la suite. Lauzon accusa Duroques d'avoir jeté un mauvais sort à sa vache ${ }^{22}$.

Les insultes où l'injurié(e) est comparé(e) à un animal tel que "bougre de chien», "cochon», «élan», "cavale», "vilaine bête» sont aussi utilisées quiclquefois (8 fois).

Enfin, il est certain que les Canadiens ont été influencés culturellement par la mère-patrie dans le choix de leurs qualificatifs injurieux. Mais on ne peut affirmer que cette influence ait été la seule car nous avons retrouvé deux cas d'insultes empruntées au contexte canadien. En 1714, la veuve Jeanne Des Colombiers de la côte Notre-Dame des Vertus, en dispute avec sa locataire Geneviève Desforges, femme de Jean-Baptiste Prévert, utilise l'expression «femme de panis ${ }^{23}$ comme insulte. En 1741 à Montréal, Angélique Bourg dit La Chapelle, pour se débarrasser du perruquier Alexandre Larchevesque qui l'importunait et voulait l'embrasser, employa le qualificatif « race de Darles» au lieu de l'expression «race de pendu», insulte que l'on entendait fréquemment en France. L'injure utilisée par la fille La Chapelle avait le même sens pour un Canadien que "race de pendu» pour un Français: ${ }^{24}$ François Darles étant ce criminel qui, le 22 août 1735 , avait été pendu sur la place publique de Montréal pour avoir incité au vol l'esclave noir Jean-Baptiste Thomas et avoir recelé les objets volés par le «noir» ${ }^{25}$. Pour ces

\footnotetext{
ANQQ, NF 25, PJN 1134.

ANQM, Documents judiciaires, avril-juin 1724, 16 au 20 juin.

ANQM, ibid., juin-décembre 1714, 6 au 19 novembre.

ANQM, ibid., septembre-décembre 1741,11 au 15 septembre.

25 ANQM, ibid., mai-août 1735, 22 août. ANQQ, NF 11-37, Conseil Supérieur, 37f., 29 v ss.
} 
deux cas qui nous restent, combien d'autres ont été perdus ou n'ont pas été traduits devant les tribunaux? Si bien qu'il est possible de croire que les Canadiens ont assez fréquemment emprunté au contexte canadien des qualificatifs injurieux et que, par conséquent, leur mentalité a été marquée par le milieu physique, social et économique dans lequel ils vivaient.

De tous ces faits divers, nous pouvons tirer quelques traits de mentalité. D'abord, le Canadien nous paraît taquin, bavard, bon vivant et conscient de ses prérogatives. L'honneur lié beaucoup ici à l'estime sociale et à la bonne réputation semble une valeur importante. Et cette valeur est d'autant plus marquante que l'on jouit, grâce à son rang social, d'une plus grande considération dans la société canadienne. C'est pourquoi nous constatons que près de $42 \%$ des plaignants sont, soit des nobles (écuyers), des officiers du roi, des marchands, soit des maîtres artisans (voir tableau III). Ils se présentent devant les tribunaux pour demander réparation pour l'offense faite à leur honneur ou à celle de leur femme ou leurs filles. Mais les gens de métier et les habitants ne sont pas en reste et

TABLEAU III

«Qualité»ou profession

Accusés

a) Domestique, apprenti

b) Journalier

c) Soldat, garde

d) Habitant

e) Gens de métier

f) Maître artisan

g) Officier de justice

h) Marchand, bourgeois

i) Écuyer, Seigneur, officier militaire

Total

Inconnue
Totaux $\% / 105$ Victimes Totaux $\% / 103$

$\begin{array}{crrr}4 & 3.8 & - & - \\ 4 & 3.8 & - & - \\ 7 & 6.6 & 5 & 4.8 \\ 32 & 30.5 & 26 & 25.2 \\ 38 & 36.2 & 29 & 28.2 \\ 4 & 3.8 & 11 & 10.7 \\ 8 & 7.6 & 13 & 12.6 \\ 3 & 2.8 & 11 & 10.7 \\ 5 & 4.8 & 8 & 7.8 \\ & & & \\ 105 & 99.9 & 103 & 100.0 \\ 42 & 28.6 & 40 & 28.0 \\ 147(1) & & 143^{(2)} & \end{array}$

1 Deux accusés avaient déclaré deux professions ou «qualités». Nous les avons retenues et comptées.

2 Une victime avait déclaré deux professions ou «qualités». Nous les avons retenues et comptées. 
veillent à ce que l'on ne porte pas atteinte à leur réputation. Ils forment $53,4 \%$ de tous les plaignants (103) dont nous connaissons la «qualité » ou profession. Et ce sont surtout des gens de métier $(36,2 \%)$ et des habitants $(30,5 \%)$ qui sont accusés de ces excès de langage. C'est ainsi que nous constatons finalement qu'il y a une tendance chez les Canadiens à intenter des poursuites judiciaires aux personnes appartenant à son groupe social. Les injures pas plus que les autres délits ${ }^{26}$ ne montrent de fortes rivalités entre les couches sociales, chacun ayant tendance à respecter l'autre et à établir des relations avec son propre groupe (relations sociales à l'horizontale). Toutefois, nous remarquons aussi chez certains groupes sociaux (domestiques, apprentis, journaliers, soldats et gens de métiers), par leurs insultes adressées aux groupes privilégiés (officiers du roi, nobles, marchands), un désir d'ascendance sociale. Bref, ce sont principalement les groupes sociaux moyens de la population canadienne que nous retrouvons devant les tribunaux pour ce type de délit. Ce sont eux qui ont le plus de difficultés à défendre leurs possessions car, faute de prestige, ils sont obligés de se faire craindre par leur intransigeance. Et cela est d'autant plus vrai qu'ils sont faciles à saisir et à dépouiller. Nous voyons paraître aussi devant les tribunaux, et en assez grand nombre, les groupes supérieurs de la population canadienne qui, à défaut de privilèges spécifiques au sein de la société canadienne, veillent à ce que les égards dus à leur rang leur soient accordés par tous. Ainsi les injures verbales, en plus d'être un instrument précieux pour retracer la mentalité du Canada du XVIII ${ }^{\mathrm{e}}$ siècle, nous permettent d'approcher la structure sociale. 